\title{
A Study of Probabilistic FEMs for a Slope Reliability Analysis Using the Stress Fields
}

\author{
Khaled Farah ${ }^{1,2, *}$, Mounir Ltifi ${ }^{1,2}$ and Hedi Hassis ${ }^{1}$ \\ ${ }^{1}$ University of Tunis El Manar, National Engineering School of Tunis, Civil Engineering Laboratory, 1002, Tunis, Tunisia \\ ${ }^{2}$ National Engineering School of Gabès, Tunisia
}

\begin{abstract}
In this paper, the applicability and the effectiveness of the probabilistic finite element methods (FEMs) such as the perturbation method, and the Spectral Stochastic Finite Element Method (SSFEM) applied to the reliability analysis of the slope stability have been studied. The results were checked by the Monte Carlo simulation and a direct coupling approach combining the deterministic finite elements code and First Order Reliability Method (FORM) algorithm. These methods are presented considering the spatial variation of soil strength parameters and Young modulus. The random field is used to describe the spatial variation. Also, the reliability analysis is conducted using a performance function formulated in terms of the stochastic stress mobilized along the sliding surface. The present study shows that the perturbation method and SSFEM can be considered as practical methods to conduct a second moment analysis of the slope stability taking into account the spatial variability of soil properties since good results are obtained with acceptable estimated relative errors. Finally, the perturbation method is performed to delimit the location of the critical probabilistic sliding surfaces and to evaluate the effect of the correlation length of soil strength parameters on the safety factor. In addition, the two methods are used to estimate the probability density and the cumulative distribution function of the factor of safety.
\end{abstract}

Keywords: FORM algorithm, monte carlo simulation, perturbation method, random field, slope stability, spatial variability.

\section{INTRODUCTION}

For a long time the engineers have analyzed the slopes stability by limit equilibrium methods. These methods have the merit to be satisfactory in spite of the simplifying assumptions on which their calculations are based. Nevertheless, several real aspects of the slope progressive failure phenomenon are ignored. Also, the uncertainties and the risks in soil properties as well as their laws of behavior are generally not taken into account in a rational manner.

Within the conventional strategy of the design, we introduce a coefficient called the coefficient of safety whose role is to cover all sources of errors and uncertainties. Concerning the problem of the slope stability, several definitions of the safety factor are proposed. The most commonly used is the ratio of the resistance moment on the moment driving of a soil mass delimited by a given slip surface. It is clear that this factor of safety does not treat the various uncertainties of the soil properties in an explicit way.

These conventional methods do not take into account the soil behavior and the distribution of the stress. The deterministic finite elements method is an alternative tool to make possible to provide indications on the soil displacement. It is able to simulate soil behavior without simplifying the problem. Also, the use of the finite element method for complex problems is necessary to obtain more reliable and accurate

*Address correspondence to this author at the National Engineering school of Tunis, Tunisia BP 37, Le Belvédère 1002 Tunis; Tel: +216 71875 726; Fax: +216 71875 726; E-mail: Khaled.Farah@ isetgf.rnu.tn results. Using the finite element method, two commonly used approaches have been developed to analyze slope stability such as gravity loading and strength reduction method. Another procedure based on stress fields had been proposed by Kim and Lee, 1997 and Farias and Naylor, 1998 [1, 2] to evaluate the safety factor and the critical slip surface. However, these methods ignore the randomness in the soil properties. To take into account these uncertainties, it is necessary to use the stochastic methods. In this context, in recent years, several probabilistic approaches have been proposed to investigate with a rational manner the slope stability problem [3-11].

The impact of uncertainties on the slope stability is often considerable. Generally speaking, the main contribution to the slope failure can be limited to the variability of soil properties. The spatial variability of the soil properties is the consequence of natural geological processes which modify continuously the soil mass [12-15]. In this work, inherent soil variability has been considered. It can be quantified by the random fields theory which represents the suitable mathematical tool [16-22]. A homogenous Gaussian random field commonly used to model the soil properties is defined by mean, variance and autocorrelation function. It has been noted that soil strength properties exhibit correlation lengths ranging from $0-6 \mathrm{~m}$ in the vertical direction and between 0 $60 \mathrm{~m}$ in the horizontal direction. The cohesion $\mathrm{c}$ and the friction angle $\phi$ are negatively cross correlated. Several formulations of stochastic finite element methods have been developed [10, 23-26], which are applied in different domains. A 
comprehensive study of the past, present and future of the stochastic finite element method has been established by Stefanou [27].

Two probabilistic methods such as perturbation method and spectral stochastic finite element method (SSFEM) applied to analyze slope stability have been studied. The methods are developed using independent random fields to describe the spatial variability of soil strength parameters and the Young modulus. Therefore, a discretization procedure is used to approximate the random fields [28-30]. Generally speaking, the probabilistic methods presented in this paper, when they are developed with random fields, their handling becomes more complex. However, the random field theory is a suitable mathematical tool commonly used to take into account the spatial variability of soil properties. Currently, in literature the majority of papers dealing with the reliability analysis of slope adopting the random field theory, homogeneous slopes are considered. For the sake of simplicity, to study the perturbation method and SSFEM method, the authors considered a homogeneous slope with no foundation layer. The different results are checked by the direct coupling approach and the Monte Carlo simulation combining a linear deterministic finite elements code. The mean and standard deviation of the safety factor are evaluated for a given critical probabilistic slip surface. The methods are used to generate the probability density function, the cumulative function of the safety factor. An investigation of the influence of the correlation length of the soil strength parameters on the safety factor is presented. In addition, the research results of the critical probabilistic circular slip surface are given considering the spatial variation of the soil strength parameters. Finally, a discussion of the applicability and the effectiveness of the methods is presented. FERUM [31] (Finite Element Reliability Using Matlab), SLOPE/W and SIGMA/W [32] coupled with programs written in MATLAB software are used to accomplish the present study.

\section{BASIC CONCEPTS}

\subsection{Accuracy of the Random field Discretization}

The Karhunen-loève discretization procedure [33] based on the spectral decomposition of its auto-covariance function is used. The discretization error can be estimated by the following relation:

$\underline{\varepsilon}=\frac{1}{\Omega_{D}} \int_{\Omega_{D}} \operatorname{srr}(x) d \Omega$

where:

$\Omega_{\mathrm{D}}$ : Random field domain.

$\operatorname{Err}(x)=\frac{\operatorname{Var}(H(x)-\widetilde{H}(x))}{\operatorname{Var}(H(x))}$

Var: Variance; and $\mathrm{H}(\mathrm{x})$ : Random field. $\widetilde{\mathrm{H}}(\mathrm{x})$ : Random field truncated at $\mathrm{N}$ terms. A very low value of $\underline{\varepsilon}$ means a high accuracy of the discretization
2.2. The Performance Function for a Slope Reliability Analysis

\subsubsection{Global Factor of Safety}

To perform a reliability analysis of a system, a performance function $\mathrm{G}$ formulated by a set of random variables $\mathrm{X}$ describing uncertainties is used. It is defined as follow:

$\mathrm{G}>0$ defines the safe domain.

$\mathrm{G}<0$ defines the failure domain.

$\mathrm{G}=0$ defines the failure surface.

In the present probabilistic analysis, the performance function $G$ is defined by the safety factor. The commonly used definition of the factor of safety can be formulated by the following relation:

$F_{s}=\frac{\int \tau_{f} d l}{\int \tau_{n} d l}$

where: $\tau_{f}$ is the shear strength determined by the Mohr Coulomb criterion;

$\tau_{n}$ is the shear stress.

$\tau_{f}=\sigma_{n} \tan \phi+c$

Using the finite element stress fields [1,2], for a given slip surface divided into $\mathrm{n}$ segments, the expression of the safety factor is:

$$
F_{S}(\xi)=\frac{\sum_{i=1}^{n}\left(c+\sigma_{i} \tan (\phi)\right) \Delta l_{i}}{\sum_{i=1}^{n} \tau_{i} \Delta l_{i}}
$$

In this equation: $\mathrm{n}$ is the number of segments of the slip surface; c is cohesion; $\phi$ is the friction angle; $\sigma_{i}$ is the normal stress; and $\tau_{i}$ is the shear stress. For a segment of the slip surface of length $\Delta l_{i}$ located in the $\mathrm{i}^{\text {th }}$ element, inclined at an angle $\alpha$ to the horizontal plan. The normal stress $\sigma_{i}$ and the shear stress $\tau_{i}$ at the middle $\mathrm{i}^{\text {th }}$ segment of the slip surface are calculated from stresses $\sigma_{\mathrm{x}}, \sigma_{\mathrm{y}}$ and $\sigma_{\mathrm{xy}}$ based on the following relations:

$$
\begin{aligned}
\tau_{i} & =0.5\left(\sigma_{y}-\sigma_{x}\right) \sin (2 \alpha)+\sigma_{x y} \cos (2 \alpha) \\
\sigma_{i} & =\sigma_{x} \sin ^{2}(\alpha)+\sigma_{y} \cos ^{2}(\alpha)-\sigma_{x y} \sin (2 \alpha)
\end{aligned}
$$

Then, the performance function used for the proposed reliability analysis can be expressed as:

$G(\xi)=F_{S}-1$

\subsubsection{Evaluation of the Stochastic Stress}

A direct determination of the stresses using the Finite Element method (FEM) at the points defining a potential surface is obtained at the gauss points. This evaluation is valid for a slip surface defined by a set of gauss points. When using a procedure to generate the slip surface, a code to interpolate the stresses is implanted. We have adopted a local smoothing method to estimate the stresses mobilized along the sliding surface. The stress at a given point $\mathrm{M}(\mathrm{x}, \mathrm{y})$ be- 
longing to a given element can be evaluated using the nodal stresses element. In the context of the finite element method, the following relation is given:

$\sigma(\xi, \eta)=\sum_{i=1}^{N e} N_{i}(\xi, \eta) \bar{\sigma}_{i}$

where : $\mathrm{N}_{i}$ is the shape function associated to the nod i, $\bar{\sigma}_{i}$ is the values of the stress at nod $\mathrm{i}, \mathrm{Ne}$ is the number of nodes selected for the local smoothing.

The approximation in the least-square sense leads to a system of equations in matrix form:

$L\left\{\sigma_{\text {noeud }}\right\}=Q\left\{\sigma_{\text {GAUSS }}\right\}$

$\sigma_{\text {GAUSS }}:$ stresses at gauss points.

The matrices $\mathrm{L}$ and $\mathrm{Q}$ are independent from the element. The stress at the given point $\mathrm{M}$ of the Cartesian coordinates is evaluated after achieving the following tasks:

1). Identify the element to which the point $\mathrm{M}$ belongs.

2). Determine the local coordinates of $\mathrm{M}$ required for evaluating the shape functions.

3). Calculate the values of element nodal stresses.

The displacements vector $\underline{\mathrm{u}}(\underline{\mathrm{x}})$ at point $\underline{\mathrm{x}}$ of elememt $\Omega_{\mathrm{e}}$ constituting the meshing are evaluated using the shape function $\mathrm{N}_{\mathrm{e}}(\underline{\mathrm{x}})$ and the vector $\mathrm{u}_{\mathrm{e}}$ of nodal displacements:

$\underline{\mathrm{u}}(\underline{\mathrm{x}})=\mathrm{N}_{\mathrm{e}}(\underline{\mathrm{x}}) \cdot \underline{\mathrm{u}_{\mathrm{e}}}$

Considering that the components of the deformations tensor are related to displacements by the matrix $\mathrm{B}(\underline{\mathrm{x}})$ deduced from derivative of the shape function by:

$\underline{\varepsilon}(\underline{x})=B(\underline{x}) \cdot \underline{u_{e}}$

In linear elasticity, the stresses are calculated by

$\underline{\sigma}(\underline{x})=D_{e} \cdot B(\underline{x}) \cdot \underline{u_{e}}$

where: $\mathrm{D}_{\mathrm{e}}$ is the elasticity matrix, $\underline{\sigma}=\left\{\begin{array}{llll}\sigma_{x} & \sigma_{y} & \sigma_{z z} & \sigma_{x y}\end{array}\right\}$

$D_{e}:$ can be written as: $D_{e}=E . D_{0}$

Where $\mathrm{D}_{0}$ constant elasticity matrix depends to the Poisson ratio.

Finally, assuming the Young modulus is a random field $E(x, \theta)$, the stochastic stress can be expressed by Eq.(14):

$\sigma(x, \theta)=E(x, \theta) D_{0} B(x) \underline{u_{e}}(\theta)$

\subsubsection{Probability Density Function and Cumulative Distri- bution Function of the Safety Factor}

The probability density function of the safety factor can be evaluated by several analyses of First Order Reliability Method (FORM). The generation of the CDF for the safety factor is summarized as follows, for different reliability indices :

1). Evaluate the two corresponding cumulative distribution functions CDF:

$$
\operatorname{CDF}= \begin{cases}\Phi(\beta) & \text { if } \mathrm{p} \geq 0.5 \\ 1-\Phi(\beta) & \text { if } \mathrm{p}<0.5\end{cases}
$$

where: $\Phi$ : Normal distribution function and $\mathrm{p}$ : Probability of failure.

2). Determine two design points $U$ on the failure surface in standard normal space

3). The independent normal random variables $U$ are mapped into a set of the basic random variables $\mathrm{X}$

4). Compute the values of $y_{i}$ by: $y_{i}=F s\left(x^{i}\right)$

Then, the probability density function $f_{F_{S}}$ is generated using the discretized cumulative distribution functions by the following relation:

$f_{F_{S}}\left(y_{i}\right)=\frac{C D F\left(y_{i}\right)-C D F\left(y_{i-1}\right)}{y_{i}-y_{i-1}}$

\section{THE PROBABILISTIC FE METHODS}

\subsection{Perturbation Method}

The perturbation method uses the Taylor series expansion of random functions about the mean values. In the context of the FEM and for quasi-static linear problems, the equilibrium is expressed as follow:

$K . U=F$

In this equation, $\mathrm{K}$ is the global stiffness matrix; $\mathrm{F}$ is the load vector and $\mathrm{U}$ is the nodal displacement vector.

The Young's modulus and the soil strength parameters are considered homogeneous random fields'. The perturbation method is formulated for $\mathrm{N}$ independent standard normal variable $\xi$ involved in the Karhunen-Loève procedure.

The second order Taylor series expansion of $K(\xi), U(\xi)$ and $F(\xi)$ can be expressed as:

$$
\begin{aligned}
& K(\xi)=K_{O}+\sum_{i=1}^{N} K_{i}^{I} \xi_{i}+\frac{1}{2} \sum_{i=1}^{N} \sum_{j=1}^{N} K_{i j}^{I I} \xi_{i} \xi_{j} \\
& U(\xi)=U_{O}+\sum_{i=1}^{N} U_{i}^{I} \xi_{i}+\frac{1}{2} \sum_{i=1}^{N} \sum_{j=1}^{N} U_{i j}^{I I} \xi_{i} \xi_{j} \\
& F(\xi)=F_{O}+\sum_{i=1}^{N} F_{i}^{I} \xi_{i}+\frac{1}{2} \sum_{i=1}^{N} \sum_{j=1}^{N} F_{i j}^{I I} \xi_{i} \xi_{j}
\end{aligned}
$$

The first and second order coefficients are obtained from the derivatives of the corresponding quantities evaluated at $\xi=0$.

By identifying the coefficient of $\xi_{i}$ and $\xi_{i} \xi_{j}$ on both sides of Eq. (16), we get:

$U_{O}=K_{O}^{-1} F_{O}$

$U_{i}^{I}=K_{O}^{-1}\left(F_{i}^{I}-K_{i}^{I} U_{O}\right)$

$U_{i}^{I I}=K_{O}^{-1}\left(F_{i j}^{I I}-K_{i}^{I} U_{i}^{I}-K_{i j}^{I I} U_{O}\right)$

Where $K_{O}, U_{O}$ and $F_{O} \quad$ are the mean values of $K, U$ and $F$, respectively. 


\subsection{Spectral Stochastic Finite Element Method (SSFEM)}

In the Spectral Stochastic Finite Element Method (SSFEM) proposed by the Ghanem and Spanos [34], the discretization Karhunen-Loève procedure is applied. Any realization of random fields $\mathrm{H}$ can be given as follows:

$H(x, \theta)=\mu(x, \theta)+\sum_{i=1}^{\infty} H_{i}(x) \xi_{i}$

where: $\xi_{\mathrm{i}}$ are standard normal random variables, $\mu(x, \theta)$ is the mean of the random field and $H_{i}(x)$ are deterministic functions that depend on the autocorrelation function. Additionally, decomposition of the nodal displacement vector solution of the problem over polynomial chaos basis $\Psi$ is used.

The truncation at $\mathrm{N}+1$ terms of the expansion stiffness matrix and at $\mathrm{P}$ terms of displacement leads to a linear system written as $K U=F$ :

$\left[\begin{array}{ccc}K_{0,0} & \cdots & K_{0,0} \\ K_{1,0} & \cdots & K_{1, P-1} \\ \vdots & & \vdots \\ K_{P-1,0} & \cdots & K_{P-1, P-1}\end{array}\right]\left[\begin{array}{c}U_{0} \\ U_{1} \\ \vdots \\ U_{P-1}\end{array}\right]=\left[\begin{array}{c}F_{0} \\ F_{1} \\ \vdots \\ F_{P-1}\end{array}\right]$

Where:

$$
\begin{array}{rl}
K_{j k}=\sum_{i=1}^{N} C_{i j k} K_{i} & k=0 \ldots P-1, C_{i j k} \\
=E\left[\xi_{i} \Psi_{j} \Psi_{k}\right] \text { and } F_{k} & =E\left[\Psi_{k} F\right]
\end{array}
$$

$K_{i}$ : are deterministic matrix obtained by assembling $k_{i}^{e}$

$k_{i}^{e}=\int_{\Omega_{e}} H_{i}\left(x, \theta_{0}\right) B^{T}(x) D_{0} B(x) d \Omega_{e}$

By solving the linear system of Eq.(24),we obtain an approximation of the displacement $\mathrm{U}(\theta)$

$U(\theta)=\sum_{k=0}^{P-1} U_{k} \Psi_{k}\left(\left\{\xi_{k}\right\}_{k=1}^{N}\right)$

where $P$ is the total number of basis polynomial chaos, $\Psi_{k}$ is the polynomial chaos.

$\mathrm{P}$ is given by

$P=\frac{(N+p) !}{N ! p !}$

Where $\mathrm{p}$ is the order of the polynomial chaos.

\subsection{Monte Carlo simulation and Direct Coupling Ap- proach}

The Monte Carlo simulation is used to generate a sample that corresponds to $\mathrm{N}$ independent standard normal variables according to the Karhunen-loève expansion of the random fields. For each realization, the factor of safety is calculated using a deterministic finite element code. The element stiffness matrix is computed for each realization of the random field $\mathrm{H}$ using the following relation:
$k^{e}\left(\theta_{0}\right)=\int_{\Omega_{e}} H\left(x, \theta_{0}\right) B^{T}(x) D_{0} B(x) d \Omega_{e}$

In this equation, $\mathrm{D}_{\alpha}$ is a constant matrix, $\mathrm{B}$ is the matrix that relates the components of strain to the nodal displacements element and $H($.$) is the random field that represents$ the soil Young's modulus. The assembling of the elements contributions Eq. (27) leads to the global stiffness matrix $K$. The Monte Carlo simulation is applied to evaluate the factors of safety, and then their statistical treatment is subsequently performed. In addition, direct coupling approach based on the combination of the deterministic finite code and FORM algorithm [30] is used to assess the reliability index. Thus, the probability of failure can be estimated. In this study, the values evaluated by the Monte Carlo simulation and direct coupling approach are considered as reference values.

A flow -chart describing the main tasks of the reliabilityanalysis by FEM is presented in Fig. (1).

\section{NUMERICAL EXAMPLE}

\subsection{The Problem Statement}

The presented probabilistic FE methods are performed to analyze the stability of a homogeneous slope with no foundation layer assuming that the mass soil is elastic. In this illustrative example, the Young's modulus and the strength parameters $\mathrm{c}$ and $\phi$ have been regarded as one-dimensional random field along the horizontal and the vertical direction. A parametric study and a search for the critical slip surfaces have been established. The statistical properties of soil parameters used are specified in Table 1. The Fig. (2) shows the boundary conditions and the slope mesh. An exponential autocorrelation function is considered. The random fields are assumed to be statistically independent. Using the stress field along the critical probabilistic slip surface, the mean, standard deviation, probability density (PDF), and cumulative distribution function (CDF) of the factor of safety could then be evaluated. Plane strain analysis is carried out.

\subsection{Preliminaries}

By neglecting the spatial variation of the soil strength parameters and the Young's modulus, the critical probabilistic slip surface is determined for correlated and uncorrelated random variables. The critical probabilistic slip surface normally corresponds to the minimum reliability index among all tested circular slip surfaces. It is used to conduct the slope reliability analysis.

In Table 2, the results of the deterministic and probabilistic approaches neglecting the spatial variation are summarized. The deterministic methods are applied using the mean values of the soil properties. The uncorrelated random variables are led to conservative results [35]. Also, the deterministic methods such as the strength reduction technique and the FEM using stress fields have provided comparable values of the factor of safely.

To reduce the computing time of the MC simulation, a sufficient number of realizations providing accurate and constant values have been found by various analyses of slope using increasing numbers of simulation. The results are 


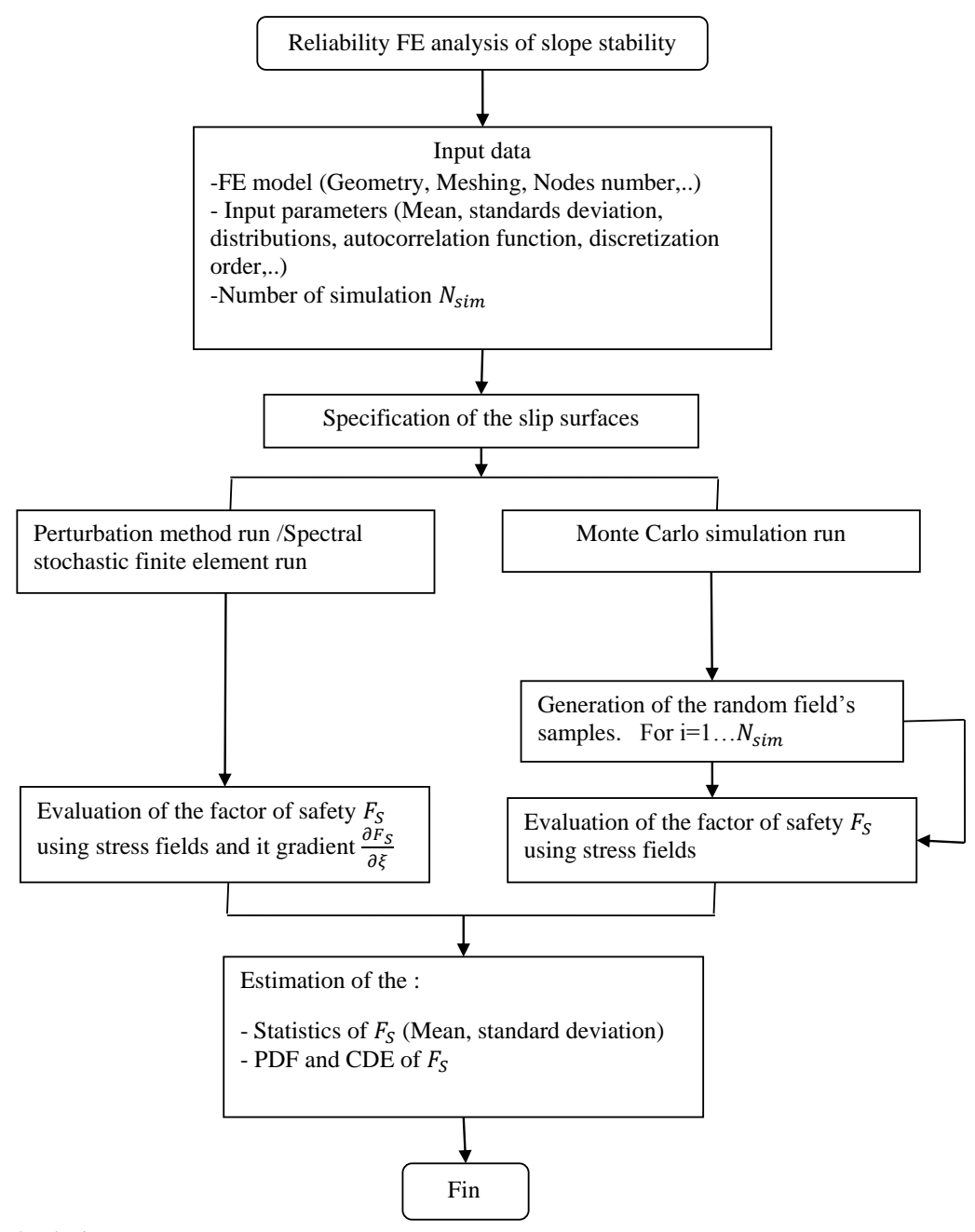

Fig. (1). Flow -chart of calculations.

Table 1. Statistical properties of soil parameters.

\begin{tabular}{|c|c|c|c|}
\hline Friction angle: $\Phi\left(^{\circ}\right)$ & 25 & 0.2 & Lognormal \\
\hline $\begin{array}{c}\text { Unit weight : } \gamma \\
\left(\mathbf{k N} / \mathrm{m}^{3}\right)\end{array}$ & 20 & ---- & ---- \\
\hline Poisson 's ratio: $v$ & 0.30 & ---- & ---- \\
\hline
\end{tabular}

shown in Figs. (3a and $\mathbf{3 b}$ ). For a number of realizations exceeding 2.500, repeatable values of the mean and standard deviation of safety factor are obtained. The Monte Carlo simulation is applied with a number of realizations equal to 3000 .

\subsection{Parametric Study}

\subsubsection{The Discretization Accuracy Influence}

The effect of the random field accuracy is investigated assuming that the cohesion $\mathrm{c}$, the friction angle $\phi$ and the
Young modulus $\mathrm{E}$ are one-dimensional homogeneous random field along the horizontal $l_{x}$ and vertical directions $l_{y}$. For different orders of the random field expansion $\mathrm{N}$, the means and standard deviation of safety factors are evaluated. The correlation lengths in the horizontal $l_{x}$ and in vertical directions $l_{y}$ are equal to $25 \mathrm{~m}$ and $2.5 \mathrm{~m}$, respectively. The results provided by the second order perturbation method, the spectral SFEM method and the Monte Carlo simulation are listed in Table 3. By considering the results of the MC simulation combining the deterministic finite element as reference, the relative errors are evaluated. 


\subsubsection{The Coefficient of Variation Influence}

The mean and the standard deviation of the safety factor are estimated for different coefficients of variation of the soil strength parameters. The correlation lengths in the horizontal and in vertical directions are held equal to $l_{x}=25 \mathrm{~m}$ and $l_{y}=2.5$, respectively. The expansion orders of the random fields are equal to $\mathrm{N}=3$. The results of the presented methods are reported in Figs. (4-7).

\subsubsection{The Correlation Length Influence}

In order to fully investigate the influence of the soil strength correlation length, we have evaluated the mean and standard deviations of the factors of safety for different correlation lengths. The random field coefficients of variation are equal to 0.2. The expansion orders of the random fields are fixed to $\mathrm{N}=4$. In Figs. (8a and $\mathbf{8 b}$ ), the results of the second order perturbation method are presented.

\subsection{The PDF and CDF of the Safety Factor}

By considering the soil strength parameters and the Young modulus are one-dimensional homogeneous random fields along the vertical direction $l_{y}$, the perturbation method and the SSFEM are performed to evaluate the probability density (PDF) and cumulative distribution function (CDF) of the factor of safety. The expansion orders of the random fields are equal to $\mathrm{N}=3$ and the order of polynomial chaos is equal to 3. Also, the correlation length is equal to $l_{y}=$ $2.5 \mathrm{~m}$. The results are presented in Figs. (9 and 10).

\subsection{The Critical Slip Surfaces}

A search for the critical circular probabilistic sliding surface is given using the probabilistic perturbation method assuming the Young modulus, friction angle and cohesion are one- dimensional random fields along the vertical direction. The direct coupling approach is performed for expansion order of the random fields equal to $\mathrm{N}=3$ and for the correlation lengths equal to $l_{y}=2.5,5$ and 1000 . The coefficients of variation of the random fields are held equal to 0.2. The results are reported in the Fig. (11). In Table 4 the minimum reliability indices estimated by the perturbation method and the direct coupling approach for different correlation lengths along the vertical direction are gathered. According to Table 4, the reliability indices evaluated by the second

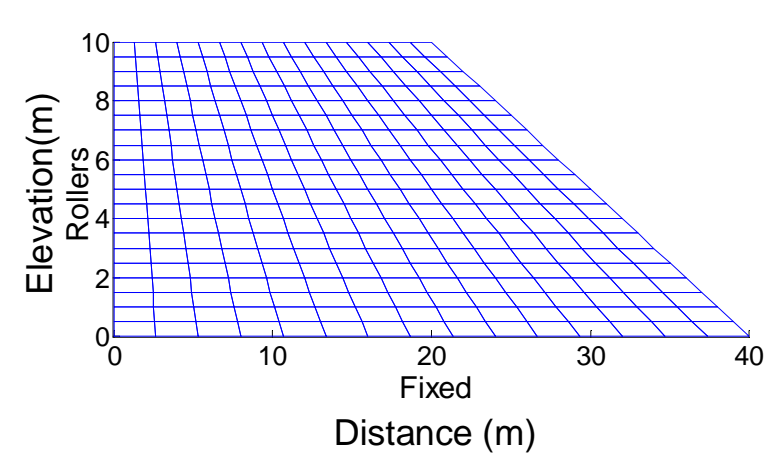

Fig. (2). The FE model of c- $\phi$ slope with no foundation layer.

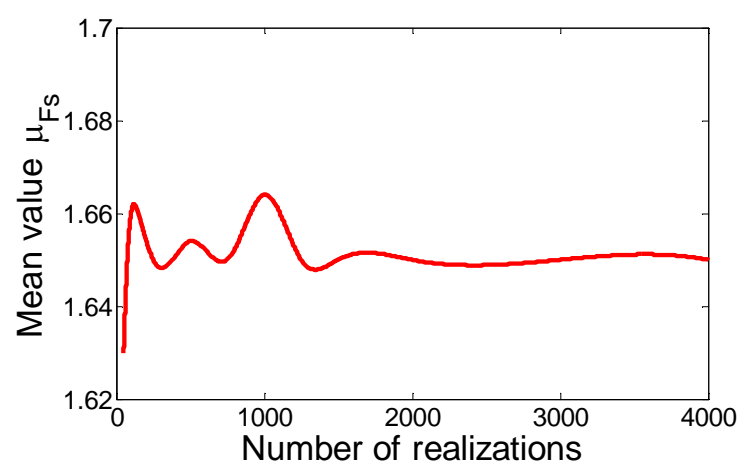

(a)

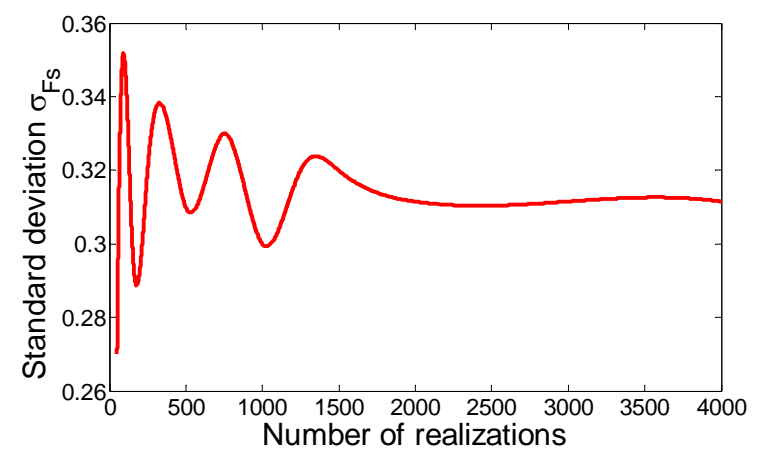

(b)

Fig. (3). Mean value ,-, $F-S$.. and standard deviation , $\sigma$-, $F-S$..by Monte Carlo simulation where the correlation lengths of the 1D Lognormal random fields in vertical direction , $1-\mathrm{y} .=2.5 \mathrm{~m}$ and $\mathrm{N}=3$ : (a) Mean value versus number of realizations, (b) Standard deviation versus number of realizations.

Table 2. Results of the deterministic and probabilistic approach neglecting the spatial variation.

\begin{tabular}{|c|c|c|c|c|c|}
\hline & & \multicolumn{2}{|c|}{ Deterministic Methods } & \multicolumn{2}{|c|}{ Probabilistic FEM Using Stress Fields } \\
\hline \multicolumn{2}{|c|}{ Reliability index $\beta$} & & --- & 2.461 & 2.944 \\
\hline \multirow{2}{*}{$\begin{array}{l}\text { The geometric character- } \\
\text { istics of slip surface }\end{array}$} & $\mathbf{R}(\mathbf{m})$ & & 31.597 & 33.702 & 33.702 \\
\hline & $y_{c}(\mathbf{m})$ & & 31.602 & 33.700 & 33.700 \\
\hline
\end{tabular}

R: radius; Circle center: $\left(x_{c}, y_{c}\right)$; FE: Finite Element Method; SRT: Strength reduction technique. 
Table 3. Influence of the random field accuracy.

\begin{tabular}{|c|c|c|c|c|c|c|c|c|c|c|c|}
\hline \multirow{2}{*}{$\begin{array}{c}\text { The Correlation } \\
\text { Length }\end{array}$} & \multirow[t]{2}{*}{$\mathbf{N}$} & \multirow[t]{2}{*}{$\underline{\boldsymbol{\varepsilon}}$} & \multicolumn{2}{|c|}{$\begin{array}{c}\text { Perturbation M. } \\
2^{\text {ieme }} \text { Order } \\
\end{array}$} & \multicolumn{3}{|c|}{ SSFEM M. } & \multicolumn{2}{|c|}{ Monte Carlo } & \multicolumn{2}{|c|}{$\begin{array}{c}\text { Relative error } \\
\%\end{array}$} \\
\hline & & & $\mu_{F}$ & $\sigma_{F}$ & $\mathbf{p}$ & $\mu_{F}$ & $\sigma_{F}$ & $\mu_{F}$ & $\sigma_{F}$ & $\mu_{F}$ & $\sigma_{F}$ \\
\hline \multirow{4}{*}{$\mathrm{l}_{\mathrm{x}}=25 \mathrm{~m}$} & 1 & 36.96 & 1.607 & 0.200 & $\begin{array}{l}1 \\
2 \\
3 \\
4\end{array}$ & $\begin{array}{l}1.550 \\
1.551 \\
1.551 \\
1.551\end{array}$ & $\begin{array}{l}0.202 \\
0.202 \\
0.202 \\
0.202\end{array}$ & 1.625 & 0.210 & -1 to -4.5 & -1 to -5 \\
\hline & 3 & 12.35 & 1.621 & 0.226 & $\begin{array}{l}1 \\
2 \\
3 \\
4\end{array}$ & $\begin{array}{l}1.551 \\
1.553 \\
1.553 \\
1.554\end{array}$ & $\begin{array}{l}0.225 \\
0.226 \\
0.226 \\
0.226\end{array}$ & 1.626 & 0.226 & $\begin{array}{l}-0.3 \text { to } \\
-4.4\end{array}$ & 0 to -0.4 \\
\hline & 6 & 5.86 & 1.620 & 0.227 & $\begin{array}{l}1 \\
2\end{array}$ & $\begin{array}{l}1.566 \\
1.567\end{array}$ & $\begin{array}{l}0.226 \\
0.227\end{array}$ & 1.627 & 0.228 & -1 to -3.7 & $\begin{array}{l}-0.4 \text { to } \\
-1\end{array}$ \\
\hline & 12 & 2.83 & ---- & --- & ---- & ---- & ---- & 1.629 & 0.230 & & \\
\hline \multirow{4}{*}{$\mathrm{l}_{\mathrm{y}}=2.50 \mathrm{~m}$} & 1 & 61.41 & 1.611 & 0.156 & $\begin{array}{l}1 \\
2 \\
3 \\
4\end{array}$ & $\begin{array}{l}1.534 \\
1.538 \\
1.538 \\
1.538\end{array}$ & $\begin{array}{l}0.151 \\
0.151 \\
0.151 \\
0.151\end{array}$ & 1.613 & 0.164 & -1 to -4.6 & -5 to -11 \\
\hline & 3 & 28.17 & 1.614 & 0.174 & $\begin{array}{l}1 \\
2 \\
3\end{array}$ & $\begin{array}{l}1.542 \\
1.545 \\
1.545\end{array}$ & $\begin{array}{l}0.173 \\
0.173 \\
0.173\end{array}$ & 1.617 & 0.174 & $\begin{array}{l}-0.2 \text { to } \\
-4.4\end{array}$ & 0 to -0.6 \\
\hline & 6 & 14.31 & 1.615 & 0.175 & $\begin{array}{l}1 \\
2\end{array}$ & $\begin{array}{l}1.554 \\
1.555\end{array}$ & $\begin{array}{l}0.174 \\
0.175\end{array}$ & 1.615 & 0.178 & 0 to -3.7 & -1 to -2 \\
\hline & 12 & 7.04 & --- & --- & --- & --- & --- & 1.616 & 0.177 & & \\
\hline
\end{tabular}

$N$ : order of the random field expansion; p:Order of the polynomial chaos

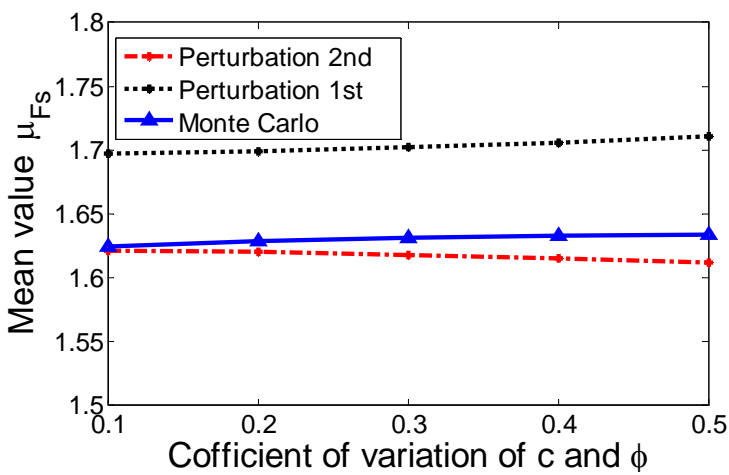

(a)

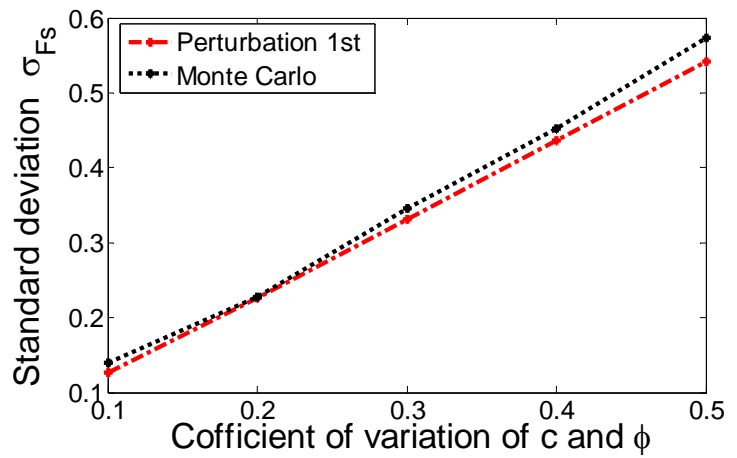

(b)

Fig. (4). Mean value $\mu_{F_{S}}$ and standard deviation $\sigma_{F_{S}}$ of the factor of safety $F_{S}$ by Perturbation method and MC simulation where the correlation lengths of the 1D Lognormal random fields in horizontal direction $\mathrm{l}_{\mathrm{x}}=25 \mathrm{~m}:$ (a) Mean value $\mu_{F_{S}}$ of $F_{S}$ versus the coefficient of variation of $\mathrm{c}$ and $\phi$ and (b) Standard deviation of $F_{S}$ versus the coefficient of variation $\sigma_{F_{S}}$ of $\mathrm{c}$ and $\phi$.

order perturbation method are close to those obtained by the direct coupling approach.

\section{DISCUSSION}

In regard to the results of this study, the following remarks can be drawn:

- The small difference between the factors of safety given in Table 2 indicates that the critical probabilistic slip surface ignoring the spatial variation of the soil strength parameters and the critical deterministic slip surface are close to each other. The result is consistent with those reported in literature [5, 36, 37]. In contrast, Fig. (11) presents the different locations of the critical sliding surfaces determined by the probabilistic perturbation method shows that, by considering the spatial variation of soil strength parameters, the critical slip surfaces varies spatially. This finding is consistent with that reported by [3]. In addition, the results presented in Table 4 indicate that taking into account spatial variability can overesti- 


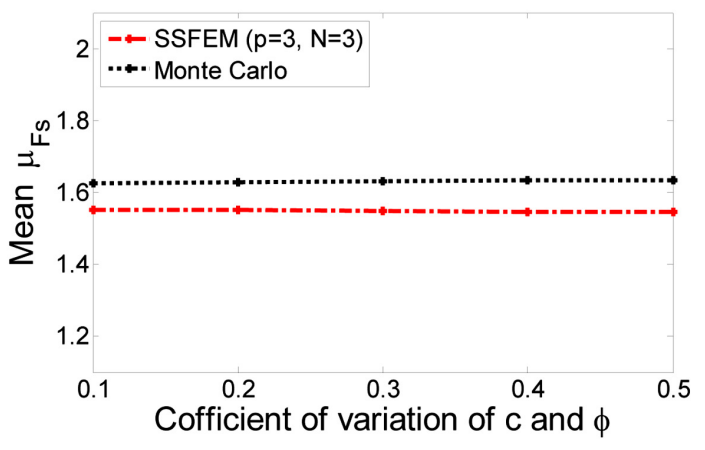

(a)

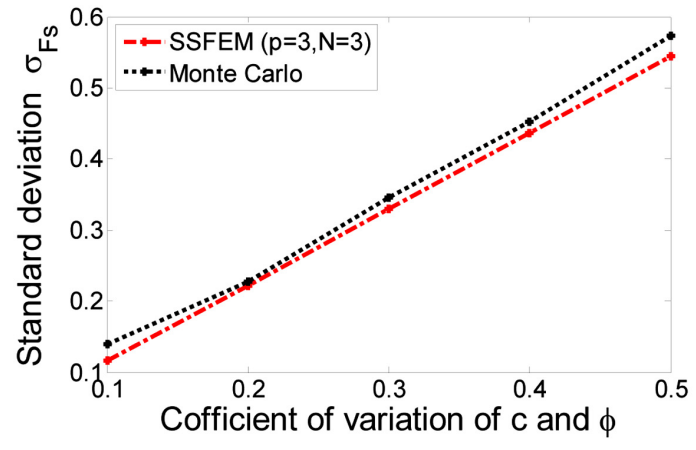

(b)

Fig. (5). Mean value $\mu_{F_{S}}$ and standard deviation $\sigma_{F_{S}}$ of the factor of safety $F_{S}$ by Perturbation method and MC simulation where the correlation lengths of the 1D Lognormal random fields in vertical direction $\mathrm{l}_{\mathrm{y}}=2.5 \mathrm{~m}:$ (a) Mean value $\mu_{F}{ }_{S}$ of $F_{S}$ versus the coefficient of variation of $\mathrm{c}$ and $\phi$ and (b) Standard deviation of $F_{S}$ versus the coefficient of variation $\sigma_{F_{S}}$ of $\mathrm{c}$ and $\phi$.

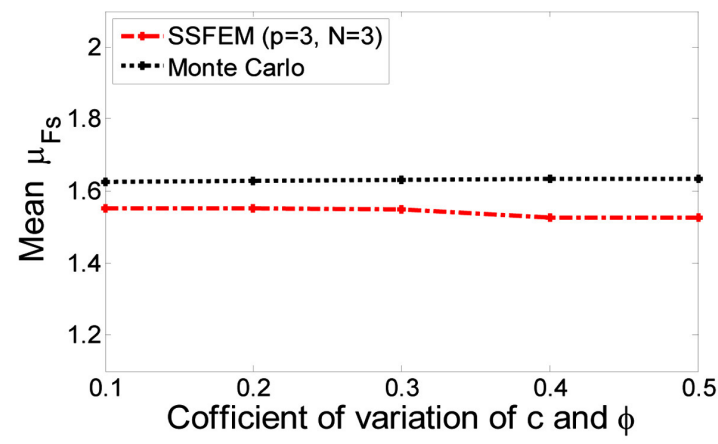

(a)

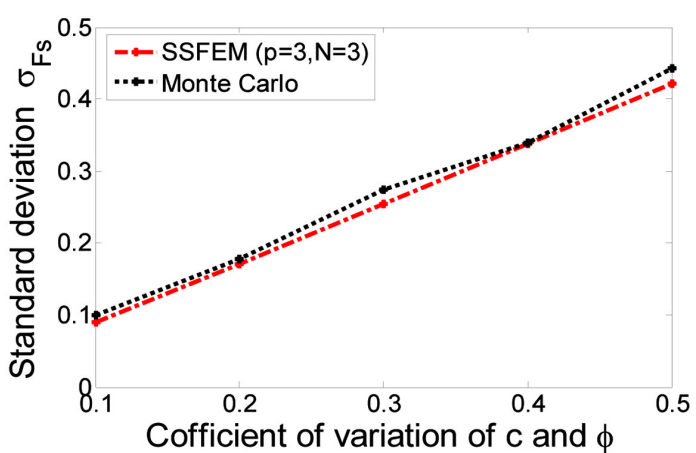

(b)

Fig. (6). Mean value $\mu_{F_{S}}$ and standard deviation $\sigma_{F_{S}}$ of the factor of safety $F_{S}$ by SSFE Method (p=3,N=3) and MC simulation where the correlation lengths of the 1D Lognormal random fields in horizontal direction $\mathrm{l}_{\mathrm{x}}=25 \mathrm{~m}$ : (a) Mean value $\mu_{F}$ of $F_{S}$ versus the coefficient of variation of $\mathrm{c}$ and $\phi$ and (b) Standard deviation of $F_{S}$ versus the coefficient of variation $\sigma_{F_{S}}$ of $\mathrm{c}$ and $\phi$.

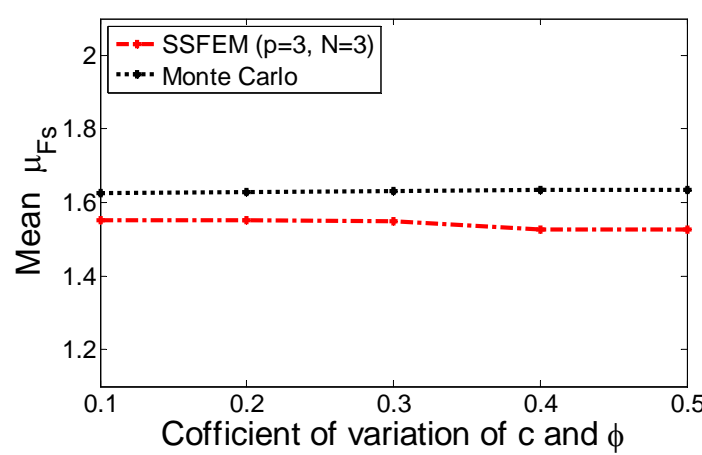

(a)

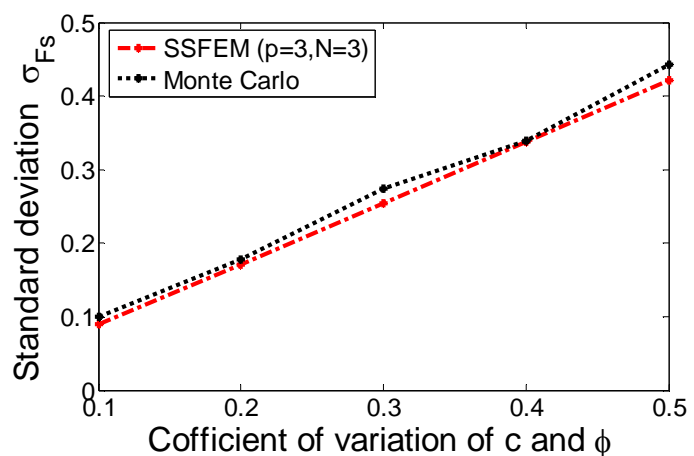

(b)

Fig. (7). Mean value $\mu_{F_{S}}$ and standard deviation $\sigma_{F_{S}}$ of the factor of safety $F_{S}$ by SSFE Method (p=3,N=3) and MC simulation where the correlation lengths of the 1D Lognormal random fields in horizontal direction $\mathrm{l}_{\mathrm{y}}=2.5 \mathrm{~m}:$ (a) Mean value $\mu_{F_{S}}$ of $F_{S}$ versus the coefficient of variation of $\mathrm{c}$ and $\phi$ and (b) Standard deviation of $F_{S}$ versus the coefficient of variation $\sigma_{F_{S}}$ of $\mathrm{c}$ and $\phi$.

mate the reliability index for low correlation lengths. Therefore, an analysis of slope stability by probabilistic methods adopting random field theory to model the spatial variability of soil strength parameters besides it is more rational than the approach neglecting the spatial variability, may lead to complementary results to those obtained by deterministic methods.

- By comparing the results listed in Table $\mathbf{3}$ obtained by the different methods to evaluate the effect of the ran- dom field accuracy on the mean and the standard deviation of the safety factor - we note that, for expansion orders $\geq 3$ which correspond to $\underline{\varepsilon}=12.35$ and $\underline{\varepsilon}=28.17$ for the horizontal and vertical directions respectivelythe random fields accuracy does not have a significant influence on the values of the means and standard deviation of the safety factor. Concerning the SSFEM, in order to reach acceptable values of the relative error of the safety factor means, it is necessary to increase the ran- 


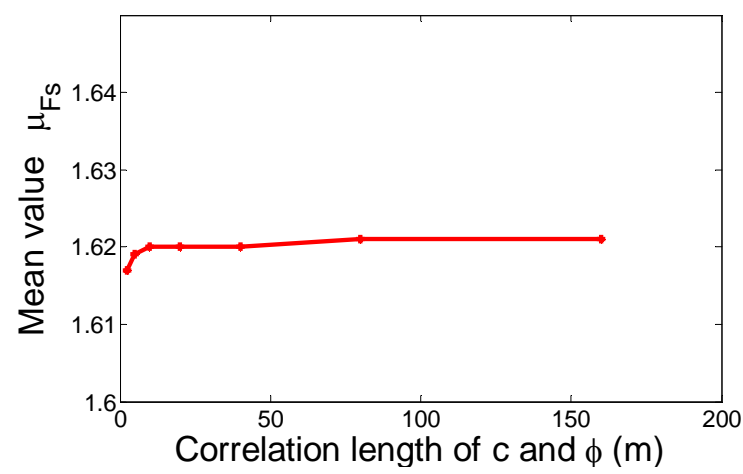

(a)

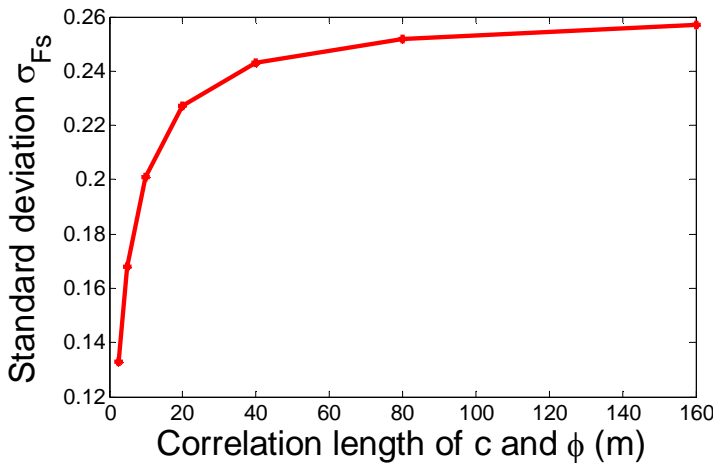

(b)

Fig. (8). The perturbation method results: the influence of the correlation length of the strength parameters on the factor of safety, the correlation length of the Young Modulus , l-y.=2.5 m.: (a) Mean, (b) Standard deviation.

dom field's expansion orders $(\mathrm{N})$ as well as those of the polynomials chaos (p). It is noted that SSFEM becomes intractable for high orders of $\mathrm{N}$ and $\mathrm{p}$. By considering the results provided by the Monte Carlo simulation as values reference, the numerical results show that the relative errors decrease when the random field's expansion orders $(\mathrm{N})$ increase. The increase of the number of the terms taken into account in the KL descritization makes possible to have precise values of the random fields. Yet, its effect on the mean and standard deviation of the safety factor remains weak for $\mathrm{N} \geq 3$. For both SSFEM and the Monte Carlo simulation applied with $\mathrm{N} \geq 3$, the mean values of the safety factor are estimated with relative errors $\leq-5 \%)$. The values of the relative errors of the standard deviations are close to all the presented methods (relative errors $\leq-2 \%$ ). Therefore, both perturbation method and SSFEM can provide satisfactory results for a second moment analysis.

- Generally speaking, although Monte Carlo simulation combined to a deterministic finite element code is too time consuming, it remains the most frequently used tool for analyzing reliability-using modeling of the spatial variability of soil properties by the random field's theory. The study performed for the determination of the simulation number has significantly reduced the computation time. In addition, Monte Carlo simulation is applied with lognormal distribution. In contrast, modeling the physical properties of a material by a Gaussian random field could give a doubtful solution due to the possible negative samples generated by the Monte Carlo simulation. However, the statistical studies of the soil properties have shown that the majority of soil properties follow the Gaussian distribution. Thus, a number of researchers have proposed stochastic finite elements formulations using Gaussian fields. They have checked the results by the Monte Carlo simulation [23, 38]. Nonetheless, in some published papers [20,39] the lognormal distribution is adopted to obtain physical values of the random fields realizations.

- Figs. (4-7) show that the results of the second orderperturbation method and the SSFEM are close to those provided by the MC simulation. Thus, they can be ap- plied to obtain good results for different values of the coefficients of variation and for small correlation length.

- The influence of the correlation length on the factor of safety is deduced from Fig. (8). It represents the mean and the standard deviation of the factor of safety for the different correlation lengths of the soil strength parameters. The standard deviation is sensitive to small values of the correlation length, they increase rapidly for $l<20 \mathrm{~m}$, becomes essentially constant for $l \geq 40 \mathrm{~m}$.

- Figs. $(\mathbf{9}, \mathbf{1 0})$ show that the probability density function (PDF) and the cumulative distribution function (CDF) of the safety factor evaluated by the SSFEM after multiple analyses of FORM are similar to those obtained by the probabilistic perturbation method.

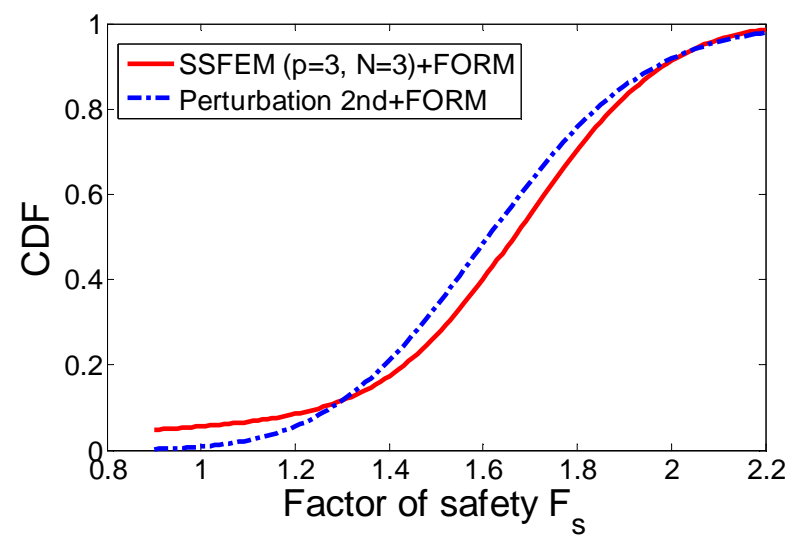

Fig. (9). Probability density functions (PDF) of the factor of safety obtained by multiple FORM analyses after SSFEM and Perturbation method.

\section{CONCLUSION}

In this paper, the perturbation method and the spectral stochastic finite element method (SSFEM) using random field theory are presented. These methods are applied to analyze the stability of a homogeneous c- $\phi$ slope assuming an elastic soil behavior. To overcome the absence of the analytical solution of the mean and standard deviation of the factor of safety, the Monte Carlo simulation combined with the deterministic finite element code is applied. The accuracy 


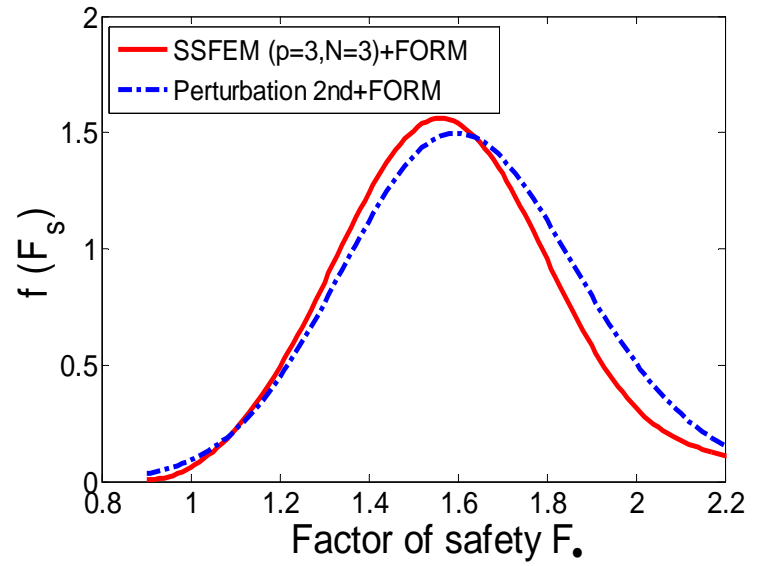

Fig. (10). Cumulative distribution functions (CDF) of the factor of safety obtained by multiple FORM analyses after SSFEM and Perturbation method.

and efficiency of the methods are evaluated by the direct coupling approach and the MC simulation.

The results of the numerical example show that the two methods can be considered suitable for the reliability analy- sis of the slope considering the spatial variability of soil strength parameters and Young modulus. In fact, the perturbation method provides satisfactory results and it is easy to apply even with high random field expansion order. Although the spectral finite element method (SSFEM) becomes intractable for high random field's expansion order, it is effective for the second moment analysis with acceptable relative errors.

Finally, the illustrative example treated in this paper has elucidated that different locations of the critical deterministic and the critical probabilistic slip surfaces are predicted when the spatial variation of the soil strength parameters and Young modulus is taken into account. Therefore, a homogeneous slope reliability analysis considering the spatial variability of soil properties has shown that a search of a probabilistic sliding area is more rational than looking for a probabilistic sliding surface.

\section{CONFLICT OF INTEREST}

The authors confirm that this article content has no conflict of interest.

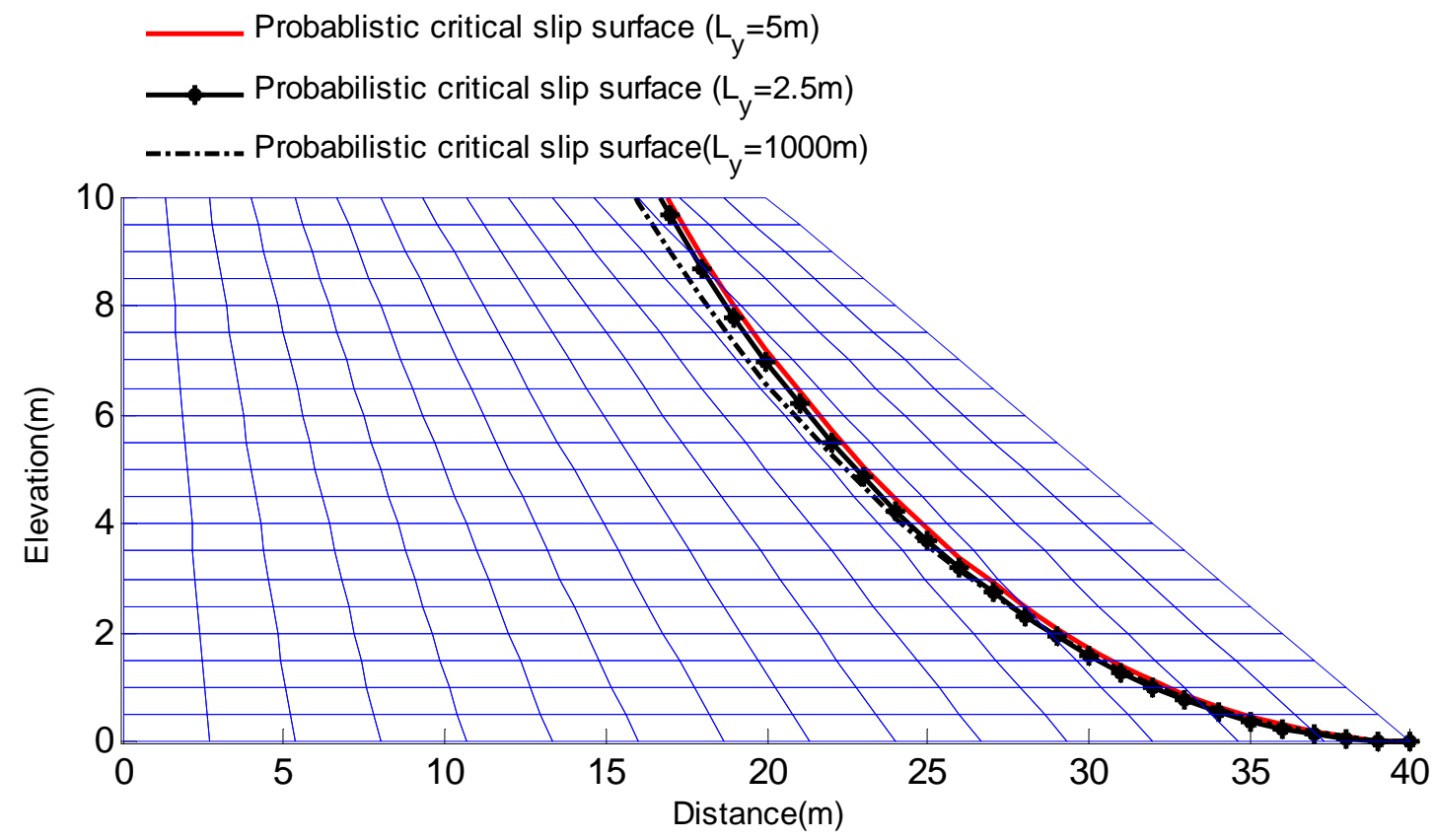

Fig. (11). Critical slip surfaces.

Table 4. Reliability indices estimated by the direct coupling approach and the perturbation method for different correlation lengths.

\begin{tabular}{|c|c|c|}
\hline \multirow{2}{*}{ Correlation length $\boldsymbol{l}_{\boldsymbol{Y}}(\mathbf{m})$} & \multicolumn{2}{|c|}{ Reliability index $\boldsymbol{\beta}$} \\
\cline { 2 - 3 } & $\begin{array}{c}\text { Perturbation M. } \\
\mathbf{2}^{\text {ieme }} \text { Order }\end{array}$ & Direct Coupling Approach \\
\hline \hline $\mathbf{2 . 5}$ & 3.793 & 3.764 \\
\hline $\mathbf{5}$ & 3.238 & 3.253 \\
\hline $\mathbf{1 0 0 0}$ & 2.455 & 2.466 \\
\hline
\end{tabular}




\section{ACKNOWLEDGEMENTS}

Declared none.

\section{REFERENCES}

[1] J.Y. Kim, and S.R. Lee, "An improved search strategy for the critical slip surface using finite element stress fields," Comput. Geotech., vol. 21, no. 4, pp. 295-313, December 1997.

[2] M.M. Farias, and D.J. Naylor, "Safety analysis using finite elements," Comput. Geotech., vol. 22, no. 2, pp. 165-181, March 1998.

[3] Y. Wang, Z. Cao, and S.K. Au, "Practical reliability analysis of slope stability by advanced Monte Carlo simulations in a spreadsheet," Can. Geotech. J., vol. 48, no. 1, pp. 162-172, January 2011.

[4] D. Li, Y. Chen, W. Lu, and C. Zhou, "Stochastic response surface method for reliability analysis of rock slopes involving correlated non-normal variables," Comput. Geotech., vol. 38, pp. 58-68, January 2011.

[5] J. Ji, H.J. Liao, and B.K. Low, "Modeling 2D spatial variation in slope reliability analysis using interpolated autocorrelations," Comput. Geotech., vol. 40, pp. 135-146, March 2012.

[6] J. Huang, A.V. Lyamin, D.V. Griffiths, K. Krabbenhoft, and S.W. Sloan, "Quantitative risk assessment of landslide by limit analysis and random fields," Comput. Geotech., vol. 53, pp. 60-67, September 2013 .

[7] P. Wang, D. Liu, H. Huang, and D. Liu, "Spoke model for calculating reliability index and safety factor of slopes," Math. Probl. Eng., vol. 2013, Article ID 829179, 7 pages, 2013. doi:10.1155/2013/ 829179.

[8] L. Li, Y. Wang, Z. Cao, and X. Chu., "Risk de-aggregation and system reliability analysis of slope stability using representative slip surfaces," Comput. Geotech., vol. 53, pp. 95-105, September 2013.

[9] A. Johari, A. Fazeli, and A.A. Javadi, "An investigation into application of jointly distributed random variables method in reliability assessment of rock slope stability," Comput. Geotech., vol. 47, pp. 42-47, January 2013.

[10] S.H. Jiang, D.Q. Li, L. M. Zhang, and C.B. Zhou, "Slope reliability analysis considering spatially variable shear strength parameters using a non-intrusive stochastic finite element method," Eng. Geol., vol. 168, pp. 120-128, January 2014.

[11] S.K. Jha, "Effect of Spatial Variability of Soil Properties on Slope Reliability Using Random Finite Element and First Order Second Moment Methods," Indian Geotech. J., pp. 1-11, April 2014.

[12] E.E. Alonso, "Risk analysis of slopes and its application to slopes in Canadian sensitive clays," Geotechnique, vol. 26, pp. 453-472, 1976.

[13] S. Lacasse, and F. Nadim, "Uncertainties in characterizing soil properties," In: C.D. Shackelford M.J.S. Roth, and P.P.Nelson, Eds., Uncertainty in Geologic Environment: From Theory to practice, Proceedings of Uncertaintainty 96, July 31-Augst3, Madison, Wisconsin, vol. 1 of Geotechnical Special Publication No.58, ASCE. New York, pp. 49-75, 1996.

[14] G.B. Baecher, and J.T. Christian, Reliability and Statistics in Geotechnical Engineering. New York: John Wiley \& Sons, 2003.

[15] K.K. Phoon, and F.H. Kulhawy, "Evaluation of geotechnical property variability," Can. Geotech. J., vol. 36, pp. 625-639, Novembr1999.

[16] E.H. Vanmarcke, "Reliability of earth slopes," J. Geotech. Eng. Division, ASCE, vol. 103, pp. 1247-1265, 1977b.

[17] H. El-Ramly, N.R. Morgenstern, and D.M. Cruden, "Probabilistic slope stability analysis of a tailings dyke on presheared clay-shale," Can. Geotech. J., vol. 40, pp. 192-208, February 2003.
[18] D.V. Griffith, and G.A. Fenton, "Probabilistic slope stability analysis by finite elements," J. Geotech. Geoenviron. Eng. Division, $A S C E$, vol. 130, no. 5, pp. 507-518, May 2004.

[19] B. Jeremić, K. Sett, and M. L. Kavvas, "Probabilistic elastoplasticity: formulation in 1D”, Acta Geotech., vol. 2, pp. 197-210, October 2007.

[20] S.E. Cho, "Effect of spatial variability of soil properties on slope stability", Eng. Geol., vol. 92, pp. 97-109, July 2007.

[21] S.E. Cho, "Probabilistic analysis of seepage that considers the spatial variability of permeability for an embankment on soil foundation," Eng. Geol., vol. 133-134, pp. 30-39, April 2012.

[22] H. Zhu, and L.M. Zhang, "Characterizing geotechnical anisotropic spatial variations using random field theory," Can. Geotech. J., vol. 50, no. 7, pp. 723-734, July 2013.

[23] M. Anders, and M. Hori, "Stochastic finite element method for elasto-plastic body," Int. J. Num. Methods Eng., vol. 46, no.11, pp. 1897-1916, December 1999.

[24] R.G. Ghanem, and P.D. Spanos, Stochastic Finite Elements: A Spectral Approach. New York: Dover publication, Mineola, 2003.

[25] R. Mellah, G. Auvinet, and F. Masrouri, "Stochastic finite element method applied to non-linear analysis of embankments," Probabilistic Eng. Mech., vol. 15, pp. 251-259, July 2000.

[26] A. Keese, and H.G. Matthies, "Efficient solvers for nonlinear stochastic problem," In: H.A. Mang, F.G. Rammmerstorfer, and J. Eberhardsteiner, Eds. Proceedings of the $5^{\text {th }}$ world Congress on computational Mechanics, July 7-12, Vienna, Austria, 2002. (http://wccm.tuwiern.ac.at/publications/Papers/fp81007.pdf,2002),

[27] G. Stefanou, "The stochastic finite element method: past, present and future," Comput. Methods. Appl. Mech. Eng., vol. 198, pp. 1031-1051, February 2009.

[28] C.C. Li, and A. Der Kiureghian, "Optimal discretization of random fields," J. Eng. Mech., vol. 119, pp. 1136-1154, June1993.

[29] M. Lemaire, Fiabilité des structures- Couplage mécano-fiabiliste statique. Paris : Hermès, 2005.

[30] B. Sudret, and A. Der Kiureghian, "Comparison of finite element reliability methods," Probabilistic Eng. Mech., vol. 17, pp. 337348 , October 2002

[31] T. Haukaas, and A. Der Kiuregian, "A computer program for nonlinear finite element analysis," In: Proceedings of the eighth international conference on structural safety and reliability, Newport Beach (CA), 2001.

[32] GEO-SLOPE International Ltd. Stability modeling with SLOPE/W 2007 Version: An engineering methodology [Computer program] GEO-SLOPE International Ltd, Calgary, Alberta, Canada.

[33] M. Loève, Probability theory. Springer Verlag : New York, $4^{\text {th }}$ ed., 1977.

[34] R.G. Ghanem, and P.D. Spanos, Stochastic finite elements - A spectral approach. New York: Springer Verlag, 1991.

[35] K.S. Li, and P. Lumb, "Probabilistic design of slopes," Can. Geotech. J., vol. 24, pp. 520-535, November 1987.

[36] A.M. Hassan, and T.F. Wolff, "Search algorithm for minimum reliability index of earth slopes", J. Geotech. Geoenviron. Eng., vol. 125, pp. 301-308, April 1999.

[37] G. Bhattacharya, D. Jana, S. Ojha, and S. Chakraborty, "Direct search for minimum reliability index of earth slopes," Comput. Geotech., vol. 30: pp. 455-462, September 2003.

[38] N.Z. Chen, and C.G. Soares, "Spectral stochastic finite element analysis for laminated composite plates," Comput. Methods Appl. Mech. Eng., vol. 197, pp. 4830-4839, October 2008.

[39] D.V. Griffiths, J. Huang, and G.A. Fenton, "Influence of spatial variability on slope reliability using 2-D random fields," J. Geotech. Geoenviron. Eng., vol. 10, pp. 1367-1378, October 2009.

This is an open access article licensed under the terms of the Creative Commons Attribution Non-Commercial License (http://creativecommons.org/licenses/ by-nc/3.0/) which permits unrestricted, non-commercial use, distribution and reproduction in any medium, provided the work is properly cited. 